\title{
Single Source of Error State Space Approach to the Beveridge Nelson Decomposition
}

\author{
Heather M. Anderson* \\ Australian National University \\ Chin Nam Low \\ Canberra, ACT, 0200 \\ AUSTRALIA \\ Monash University \\ Clayton, Victoria 3800 \\ AUSTRALIA \\ Ralph Snyder \\ Monash University \\ Clayton, Victoria 3800 \\ AUSTRALIA
}

June 7, 2004

\begin{abstract}
A bstract
The use of the Beveridge Nelson decomposition in macroeconomic analysis involves the truncation and estimation of infinite weighted sums of random variables. The single source of error state space approach provides a simple and effective alternative that leads to exactly the same decomposition without the infinite weighted sum and the required truncation. As such it provides a useful macroeconomic tool that simplifies the calculation of the relative importance of permanent and temporary shocks.
\end{abstract}

Keywords: Single source of error; Beveridge Nelson decomposition; state-space models.

JEL classification: C22, C51, E32

*Correspondence: E-mail: Heather.Anderson@anu.edu.au 


\section{Introduction}

Two stylised facts associated with most macroeconomic time series are that they exhibit long run growth and recurrent fluctuations around the growth path. This has often led to exercises which decompose macroeconomic series into trend and cyclical components, where the trend represents long run growth in the economy and the cycle represents the business cycle. There are many different ways in which this decomposition is undertaken (see Canova (1998) for a recent survey), and there is considerable debate about which decomposition (if any) leads to trends and cycles that best capture the features that economists typically associate with economic growth and business cycles.

One decomposition that has attracted considerable attention in the applied macroeconomics literature is the one first proposed by Beveridge and Nelson (BN) (1981). They defined the trend of an ARIMA $(p, 1, q)$ series as the level of the long run forecast of a series (minus the deterministic trend, if any), and the cycle as the difference between the present level and the trend component. This decomposition is based on forecasting considerations, because not only does the $\mathrm{BN}$ trend embody the (time $t$ ) long run forecast of the series, but the BN cycle also embodies the forecastable momentum of the series at each point in time. A by-product of the $\mathrm{BN}$ decomposition is that the innovations in the trend and the cyclical components are perfectly (and often negatively) correlated, which allows for the possibility that the BN trend and the BN cycle are driven by the same innovation.

The forecasting literature has a long tradition of decomposing time series into trends and cycles, and like the macroeconomic literature, there are various ways in which this decomposition is undertaken and debate about which way is best. One popular decomposition that is often used in forecasting is the unobserved components (UC) decomposition advocated by Harvey (1985), in which the innovations in the trend and cyclical components have zero correlation by assumption. Watson (1986), Stock and Watson (1988), and Harvey and Koopman (2000) explore some of the properties of this decomposition. An alternative forecasting approach introduced by Ord, Koehler and Snyder (1997) is the class of state space models with a single source of disturbance. In these latter models, the innovations of the unobserved state components as well 
as the observations are all perfectly correlated, because they are driven by the same disturbance. It is this similarity with the BN property that motivates the use of a single source of error (SSOE) state space forecasting approach to estimate the trend and cycle components of $\mathrm{BN}$ decomposition.

Harvey and Koopman (2000) have observed that the BN trend and cycle components for an $\operatorname{ARIMA}(0,1,1)$ model correspond to those from the UC decomposition with perfectly correlated disturbances. Here, we generalise this observation to point out that the SSOE state space forecasting approach can be used to obtain the BN trend and cycle components for any series with a $\operatorname{ARIMA}(p, 1, q)$ process. Previous literature, including Miller (1988), Newbold (1990) and Morley (2002) has noted the difficulties involved with trucating and estimating the infinite sums in the $\mathrm{BN}$ trend, and has proposed various computational methods to overcome this problem. The main advantage of the SSOE state space forecasting approach is that in addition to providing an easy way to avoid the truncation of the infinite sum in the trend component, it also allows a very straight-forward comparison of the variances of the trend and cycle innovations.

\section{Beveridge $\mathrm{N}$ elson Decomposition}

Assume that $y_{t}$ is a $\mathrm{I}(1)$ variable with a Wold representation given by

$$
\Delta y_{t}=\mu+\gamma(L) \varepsilon_{t}
$$

where $\mu$ is the long run growth or drift, $\gamma(L)$ is a polynomial in the lag operator $L$ with $\gamma(0)=1$ and $\Sigma_{i=0}^{\infty}\left|\gamma_{i}\right|<\infty$, and $\varepsilon_{t}$ is an iid $\left(0, \sigma^{2}\right)$ one-step-ahead forecast error of $y_{t}$. The process $\gamma(L) \varepsilon_{t}$ can be approximated as an $\operatorname{ARMA}(p, q)$ process, with $\gamma(L)=\frac{\theta(L)}{\phi(L)}$, $\theta(L)=1+\theta_{1} L+\theta_{2} L^{2} \ldots \theta_{q} L^{q}$ and $\phi(L)=1+\phi_{1} L+\phi_{2} L^{2} \ldots \phi_{p} L^{p}$.

The $h$ step-ahead forecast of $y_{t}$ (denoted by $\widehat{y}_{t+h}$ ) is given by

$$
\widehat{y}_{t+h}=\mu h+y_{t}+\left(\sum_{i=1}^{h} \gamma_{i}\right) \varepsilon_{t}+\left(\sum_{i=2}^{h+1} \gamma_{i}\right) \varepsilon_{t-1}+\ldots \ldots \ldots
$$

For large values of $h, \mathrm{BN}$ (1981) approximated the $h$ step-ahead forecast as

$$
\widehat{y}_{t+h} \approx \mu h+y_{t}+\left(\Sigma_{i=1}^{\infty} \gamma_{i}\right) \varepsilon_{t}+\left(\Sigma_{i=2}^{\infty} \gamma_{i}\right) \varepsilon_{t-1}+\ldots \ldots \ldots .
$$


This shows that the long run forecast is asymptotic to a linear function (with slope $\mu$ ) of the forecast horizon $h$, and a stochastic intercept (which is often called 'the level').

BN (1981) used the 'level' at time $t$ of the long run forecast given by equation (2.3) to define the trend $\tau_{t}$ by

$$
\tau_{t}=y_{t}+\left(\sum_{i=1}^{\infty} \gamma_{i}\right) \varepsilon_{t}+\left(\sum_{i=2}^{\infty} \gamma_{i}\right) \varepsilon_{t-1}+\ldots \ldots
$$

Taking the first difference in equation (2.4) we have

$$
\tau_{t}-\tau_{t-1}=\Delta y_{t}+\left(\sum_{i=1}^{\infty} \gamma_{i}\right) \varepsilon_{t}-\left(\gamma_{1} \varepsilon_{t-1}+\gamma_{2} \varepsilon_{t-2}+\ldots . .\right),
$$

which in view of equation (2.1) for $\Delta y_{t}$ reduces to

$$
\tau_{t}-\tau_{t-1}=\mu+\gamma(1) \varepsilon_{t}
$$

with $\gamma(1)=\Sigma_{i=0}^{\infty} \gamma_{i}$ and $\gamma_{0}=1$. Hence, the trend component $\tau_{t}$ is a random walk with drift equal to $\mu$ and a non-autocorrelated innovation given by $\gamma(1) \varepsilon_{t}$.

The cyclical component $c_{t}$ is defined as the difference between the current level $y_{t}$ and the trend $\tau_{t}$. From equations (2.1) and (2.6), $c_{t}$ is given by

$$
c_{t}=\left(\frac{\gamma(L)-\gamma(1)}{(1-L)}\right) \varepsilon_{t}=\left(\frac{\theta(L)-\gamma(1) \phi(L)}{\phi(L)(1-L)}\right) \varepsilon_{t}=\frac{\psi(L)}{\phi(L)} \varepsilon_{t},
$$

where $\psi_{0}=1-\gamma(1)$, and the order of $\psi(L)$ is $n$ with $n \leq \max (p-1, q-1)$ (due to the unit root in $\theta(L)-\gamma(1) \phi(L))$. It is clear from equations (2.6) and (2.7) that the trend and cycle components are driven by the same innovation, so that the innovations to the trend and cycle are perfectly correlated.

The truncation of the infinite sum in equation (2.4) complicates the estimation of the trend and cyclical components. BN evaluated their expression for $\tau_{t}$ by setting $h$ equal to a suitably large positive integer, while Newbold (1990) and Miller (1988) have derived alternative expressions for $\tau_{t}$ to simplify their calculations of $\tau_{t}$. Morley (2002) uses a state space approach to simplify the decomposition, but he doesn't use the perfect correlation between $\tau_{t}$ and $c_{t}$ to parameterise his state space model. 


\section{Single Source of Error State Space M odels}

The linear single source of error state space model proposed by Snyder (1985) is given by

$$
y_{t}=\beta^{\prime} x_{t-1}+e_{t}
$$

and

$$
x_{t}=F x_{t-1}+\alpha e_{t}
$$

where (3.1a) is known as the measurement equation and (3.1b) is known as the system equation. The $k$ vector $x_{t}$ represents the unobserved state of the underlying process at the beginning of period $t, \alpha$ is a fixed $k$ vector of parameters, $e_{t}$ is an iid $\left(0, \sigma^{2}\right)$ innovation, $\beta$ is a fixed $k$ vector, and $F$ is a fixed $k \times k$ transition matrix. Often both $\beta$ and $F$ depend on a set of time invariant parameters. The key feature of this specification is that both equations are driven by the same innovation.

Snyder (1985) shows that the likelihood function associated with (3.1a) and (3.1b) is very simple, so that it is convenient to obtain maximum likelihood estimates of the parameters using the prediction error decomposition of the likelihood in conjunction with a suitable version of the Kalman filter. Further details relating to estimation are described in Snyder (1985) or Harvey (1989).

The above state space model is stable if the matrix $\left(F-\alpha \beta^{\prime}\right)$, also known as the discount matrix, has eigenvalues with absolute value less than one (Ord, Koehler, and Snyder (1997)). Letting $\left(F-\alpha \beta^{\prime}\right)=D$, it can be shown that

$$
x_{t}=\sum_{j=0}^{\infty} D^{j} \alpha y_{t-j}
$$

and

$$
y_{t}=\sum_{j=1}^{\infty} \beta^{\prime} D^{j} \alpha y_{t-j}+e_{t}
$$

Hence, when $D$ is strictly stable, $D^{j} \longrightarrow 0$ when $j \longrightarrow \infty$, the past observations have a declining effect as one moves further back in time. Equation (3.3) resembles Blanchard's (1979) "backwards" solution to a rational expectations model. 


\section{Single Source of Error State Space A pproach to BN Decomposition}

Consider a time series $y_{t}$ with an approximated $\operatorname{ARIMA}(p, 1, q)$ process represented by (1). The I(1) term allows the series to be broken down into its trend $\left(\tau_{t}\right)$ and cycle $\left(c_{t}\right)$ components in accordance with the $\mathrm{BN}$ decomposition so that

$$
y_{t}=\tau_{t}+c_{t}
$$

with

$$
\tau_{t}=\mu+\tau_{t-1}+\alpha \varepsilon_{t} \text { and } c_{t}=\frac{\psi(L)}{\phi(L)} \varepsilon_{t},
$$

where $\alpha=\gamma(1)$ from equation (2.6), $\psi_{0}=1-\alpha$ and the order of $\psi(L)$ is $n$ with $n \leq \max (p-1, q-1)$ (see (equation 2.7)).

Substituting equations (4.2) into (4.1) gives

$$
y_{t}=\mu+\tau_{t-1}+\alpha \varepsilon_{t}-\phi_{1} c_{t-1}-\phi_{2} c_{t-2}-\ldots . \phi_{p} c_{t-p}+\psi_{0} \varepsilon_{t}+\psi_{1} \varepsilon_{t-1}+\ldots . .+\psi_{n} \varepsilon_{t-n},
$$

which reflects the intuition that since $y_{t}$ comprises of two components (i.e. the trend $\left(\tau_{t}\right)$ and cycle $\left.\left(c_{t}\right)\right)$, the one-step-ahead forecast error $\varepsilon_{t}$ of output should equal the sum of the forecast errors of the two components, which are $\alpha \varepsilon_{t}$ and $(1-\alpha) \varepsilon_{t}$ respectively.

Letting $\phi_{p}^{*}(L)=-\phi_{1} L-\phi_{2} L^{2} \ldots-\phi_{p} L^{p}$ and $\psi_{n}^{*}(L)=\psi_{1} L+\psi_{2} L^{2} \ldots .+\psi_{n} L^{n}$, the measurement equation for the single source of error state space approach can be written as

$$
y_{t}=\mu+\tau_{t-1}+\phi_{p}^{*}(L) c_{t}+\psi_{n}^{*}(L) \varepsilon_{t}+\varepsilon_{t}
$$

and the state or transition equations are

$$
\tau_{t}=\mu+\tau_{t-1}+\alpha \varepsilon_{t}
$$

and

$$
c_{t}=\phi_{p}^{*}(L) c_{t}+\psi_{n}^{*}(L) \varepsilon_{t}+(1-\alpha) \varepsilon_{t} .
$$

The formulation of equations (4.4b) and (4.4c), which form the state transition equation in (3.1b), are somewhat similar to the UC decompositions in Watson (1986), Stock and Watson (1988), and Harvey and Koopman (2000). However, a critical difference 
is that the two equations are driven by the same innovation and are perfectly correlated, unlike the UC decomposition in which the trend and cycle disturbances have zero correlation.

In practice, if $\alpha<1$ then the trend and cycle will have perfect positive correlation and both components will share in the variation of the data. However, if $\alpha>1$, then the innovations in the trend and cycle will have perfect negative correlation, and the trend will generate most of the variation in the data since $|\alpha|$ will then be greater than $|1-\alpha|$. Morley, Nelson and Zivot (2003) observed this latter situation for real US GDP.

\section{Applications}

We illustrate the use of the single source error state space approach to compute the Beveridge Nelson trend/cycle decompositions for $\operatorname{ARIMA}(0,1,1), \operatorname{ARIMA}(1,1,0)$ and $\operatorname{ARIMA}(2,1,2)$ models of the logarithms of real output for the United States, the United Kingdom and Australia. The US models coincide with those used by Stock and Watson (1988) in their study of the importance of the trend component in real US GNP, and we broaden the scope to include decompositions for UK and Australia to demonstrate the relative importance of trends in other countries. We use quarterly GNP data for the USA (from 1947:1 to 2003:1), and quarterly GDP data for the UK and Australia (from 1960:1 to 2003:1). Our parameter of interest is $\alpha$, which is Campbell and Mankiw's (1987) persistence measure that predicts the long run increase in GNP/GDP resulting from a $1 \%$ shock in GNP/GDP in one quarter. Since researchers are often interested in the fraction of the variance in the quarterly change in real output that can be attributed to changes in its stochastic trend, we use our computed BN trends to calculate Stock and Watson's (1988) $R^{2}$ measure of this ratio. The empirical results are presented in Table 1, and we outline details relating to the SSOE state space formulation below.

\subsection{ARIM A $(0,1,1)$ model}

The BN trend and cycle components for an $\operatorname{ARIMA}(0,1,1)$ model are well known to be

$$
\tau_{t}=\mu+\tau_{t-1}+\alpha \varepsilon_{t} \text { and }
$$




$$
c_{t}=(1-\alpha) \varepsilon_{t},
$$

where, in terms of the ARMA coefficients for $\Delta y_{t}, \alpha=\gamma(1)=1+\theta_{1}$. These equations can be cast into single source of error state space form with

$$
y_{t}=\mu+\left[\begin{array}{ll}
1 & 0
\end{array}\right]\left[\begin{array}{c}
\tau_{t-1} \\
c_{t-1}
\end{array}\right]+\varepsilon_{t}
$$

as the measurement equation and

$$
\left[\begin{array}{l}
\tau_{t} \\
c_{t}
\end{array}\right]=\left[\begin{array}{l}
\mu \\
0
\end{array}\right]+\left[\begin{array}{ll}
1 & 0 \\
0 & 0
\end{array}\right]\left[\begin{array}{c}
\tau_{t-1} \\
c_{t-1}
\end{array}\right]+\left[\begin{array}{c}
\alpha \\
1-\alpha
\end{array}\right] \varepsilon_{t} .
$$

Forecasts for these state space equations can be computed by using a suitable version of the Kalman filter and the maximum likelihood estimates of the parameters obtained using the prediction error decomposition of the likelihood function The eigenvalues of the discount matrix $\left(F-\alpha \beta^{\prime}\right)$ (from equation 8) need to be within the unit circle to ensure stability, and this condition is satisfied for each of the three decompositions undertaken here.

The estimated $\alpha$ s and implied variance ratios for USA, UK and Australian output are shown in Table1. Here it is interesting to note that while $\alpha>1$ for the USA, implying that innovations to the trend and cycle are negatively correlated in this case, the same is not true for the UK and Australia. Turning to the $R^{2}$ measures of the fraction of the variance in the quarterly change in real output that can be attributed to changes in its stochastic trend, we see that trend makes a relatively lower contribution in the USA, than it does in the UK and Australia.

\subsection{ARIMA $(1,1,0)$ model}

For an $\operatorname{ARIMA}(1,1,0)$ model the trend component is the same as above, although in this case $\alpha=\frac{1}{1+\phi_{1}}$ in terms of the ARMA coefficients for $\Delta y_{t}$. The cycle component is given by

$$
c_{t}=-\phi_{1} c_{t-1}+(1-\alpha) \varepsilon_{t} .
$$


Arranging the model into state space form, the measurement equation is

$$
y_{t}=\mu+\left[\begin{array}{ll}
1 & -\phi_{1}
\end{array}\right]\left[\begin{array}{c}
\tau_{t-1} \\
c_{t-1}
\end{array}\right]+\varepsilon_{t}
$$

and the transition equation is

$$
\left[\begin{array}{c}
\tau_{t} \\
c_{t}
\end{array}\right]=\left[\begin{array}{l}
\mu \\
0
\end{array}\right]+\left[\begin{array}{cc}
1 & 0 \\
0 & -\phi_{1}
\end{array}\right]\left[\begin{array}{c}
\tau_{t-1} \\
c_{t-1}
\end{array}\right]+\left[\begin{array}{c}
\alpha \\
1-\alpha
\end{array}\right] \varepsilon_{t} .
$$

Estimation of the state space model imposes the identity that $\phi_{1}=\frac{1-\alpha}{\alpha}$ (which arises from the observation that $\alpha=\frac{1}{1+\phi_{1}}$ ), and the results are reported in Table 1 . As above, appropriate stability conditions (in terms of the eigenvalues for the discount matrix) are satisfied for each country. The results are very similar to those for the $\operatorname{ARIMA}(0,1,1)$ model in that $\alpha>1$ for the USA, while $\alpha<1$ for the UK and Australia. Also, the implied $R^{2}$ for the USA is much smaller than those for the UK and Australia, reflecting comparitively less noisy cycles in the latter countries.

\subsection{ARIMA $(2,1,2)$ model}

The ARIMA $(2,1,2)$ model of output has been used by Morley, Nelson and Zivot (2003) for US GDP, and it is the model that is chosen using standard model selection criteria for the USA and Australia. As usual, the trend component is given by equation (4.2), while the cycle component is given by

$$
c_{t}=-\phi_{1} c_{t-1}-\phi_{2} c_{t-2}+\theta_{1} \varepsilon_{t-1}+(1-\alpha) \varepsilon_{t} .
$$

In this case $\alpha=\frac{1+\theta_{1}+\theta_{2}}{1+\phi_{1}+\phi_{2}}$ in terms of the ARMA coefficients for $\Delta y_{t}$, although this relationship does not affect the following estimation.

The model can be cast into a single source of error state space form with

$$
y_{t}=\mu+\left[\begin{array}{llll}
1 & -\phi_{1} & -\phi_{2} & \theta_{1}
\end{array}\right]\left[\begin{array}{c}
\tau_{t-1} \\
c_{t-1} \\
c_{t-2} \\
\varepsilon_{t-1}
\end{array}\right]+\varepsilon_{t}
$$


being the measurement equation, and

$$
\left[\begin{array}{c}
\tau_{t} \\
c_{t} \\
c_{t-1} \\
\varepsilon_{t}
\end{array}\right]=\left[\begin{array}{l}
\mu \\
0 \\
0 \\
0
\end{array}\right]+\left[\begin{array}{cccc}
1 & 0 & 0 & 0 \\
0 & -\phi_{1} & -\phi_{2} & \theta_{1} \\
0 & 1 & 0 & 0 \\
0 & 0 & 0 & 0
\end{array}\right]\left[\begin{array}{c}
\tau_{t-1} \\
c_{t-1} \\
c_{t-2} \\
\varepsilon_{t-1}
\end{array}\right]+\left[\begin{array}{c}
\alpha \\
1-\alpha \\
0 \\
1
\end{array}\right] \varepsilon_{t}
$$

being the transition equation.

Estimated results are presented in Table 1 below. As above, appropriate stability conditions (in terms of the eigenvalues for the discount matrix) are satisfied for US and UK and very close to unity for Australia. In the case of the UK real GDP, some of the estimated parameters are insignificantly different to zero. The UK cycle component in equation (5.5) is simplified to its parsimonious $\operatorname{ARIMA}(2,1,2)$ form by making $\theta_{1}=$ 0 , removing the MA term. The overall results are very similar to those found for the $\operatorname{ARIMA}(0,1,1)$ and $\operatorname{ARIMA}(1,1,0)$ models, although the estimated $\alpha$ for Australia and the UK is now greater than one. Once again, the results suggest that the trend component in the US decomposition is relatively less important than the corresponding trends in the UK and Australian decompositions.

\section{Conclusion}

In this paper a single source of error state space approach has been proposed to exactly compute the trend and cyclical components of the $\mathrm{BN}$ decomposition in accordance with the original BN property that the two components are perfectly correlated. This approach offers a simple and straight forward formulation of both the trend and cyclical components in state space form to fit the required ARIMA model. It also highlights and confirms that the forecast error is solely contributed by the correlated innovations of these two components and hence, provides a basis to analyse the dynamics between the trend and cycle in the data generating process which is driven by the value of the long run multiplier $\alpha$. 
Table 1

M easures of the importance of trend in real log GNP/GDP

\begin{tabular}{|c|c|c|}
\hline $\begin{array}{l}\text { Univariate } \\
\text { Statistical } \\
\text { Model }\end{array}$ & $\begin{array}{l}\text { Long-run change in GNP } \\
\text { predicted from a } 1 \% \text { shock change } \\
\text { in GNP in one quarter }\left(\alpha^{*}\right)\end{array}$ & $\begin{array}{c}\text { Variance ratios } \\
\mathrm{R}^{2}\end{array}$ \\
\hline US GNP & Data from 1947:I to 2003:I & \\
\hline $\operatorname{ARIMA}(0,1,1)$ & $\begin{array}{l}1.2701 \\
(0.0552)^{1}\end{array}$ & 0.9339 \\
\hline $\operatorname{ARIMA}(1,1,0)$ & $\begin{array}{l}1.5226 \\
(0.0 .1464)\end{array}$ & 0.8817 \\
\hline $\operatorname{ARIMA}(2,1,2)$ & $\begin{array}{l}1.2653 \\
(0.1459) \\
\end{array}$ & 0.8458 \\
\hline UK GDP & Data from 1960:I to 2003:I & \\
\hline $\operatorname{ARIMA}(0,1,1)$ & $\begin{array}{l}0.9945 \\
(0.0724)\end{array}$ & 0.9999 \\
\hline $\operatorname{ARIMA}(1,1,0)$ & $\begin{array}{l}0.9940 \\
(0.0759)\end{array}$ & 0.9999 \\
\hline $\operatorname{ARIMA}(2,1,2)$ & $\begin{array}{l}1.2267 \\
(0.1587)\end{array}$ & 0.9686 \\
\hline Australia GDP & Data from 1960:I to 2003:I & \\
\hline $\operatorname{ARIMA}(0,1,1)$ & $\begin{array}{l}0.9594 \\
(0.0758)\end{array}$ & 0.9983 \\
\hline $\operatorname{ARIMA}(1,1,0)$ & $\begin{array}{l}0.9604 \\
(0.0702)\end{array}$ & 0.9983 \\
\hline $\operatorname{ARIMA}(2,1,2)$ & $\begin{array}{l}1.0394 \\
(0.0356) \\
\end{array}$ & 0.9552 \\
\hline
\end{tabular}

$1_{\text {std. error in parenthesis }}$

* estimates of $\alpha$. Estimates of other coefficients can be requested from authors

The $\mathrm{R}^{2}$ statistic is obtained by regressing the quarterly change in GNP against the change in the BN trend 


\section{R eferences:}

Beveridge, S., and C. R. Nelson (1981). "A New Approach to Decomposition of Economic Time Series into Permanent and Transitory Components with Particular Attention to Measurement of the Business Cycle". J ournal of Monetary Economics, 7, 151-174.

Blanchard, O. (1979). "Backward and Forward Solutions for Economies with Rational Expectations", The American E conomic Review, 114 - 118.

Campbell, J. and N.G.Mankiw (1987). "Permanent and Transitory Components in Macroeconomic Fluctuations", The American E conomic Review, 77, 111-117.

Canova, F. (1998). "Detrending and Business Cycle Facts," J ournal of Monetary E conomics, 41, 475 - 512.

Harvey, A. C. (1989). "Forecasting, Structural Time Series M odels and the Kalman Filter". Cambridge University Press.

Harvey, A.C., and S. J. Koopman (2000). "Signal Extraction and the Formulation of Unobserved Components Models". The E conometrics J ournal, Vol 3, 84-107.

Miller, M. (1988). "The Beveridge-Nelson Decomposition of Economic Time Series - Another Economical Computational Method", J ournal of M onetary Economics, 21, 141-142.

Morley, J. C. (2002). "A State-Space Approach to Calculating the Beveridge-Nelson Decomposition", E conomics Letters, 75, 123-127.

Morley, J. C., C. R. Nelson, and E. Zivot (2003). "Why are the Beveridge-Nelson and Unobserved Components Decompositions of GDP so Different?", The Review of E conomics and Statistics, Vol 85(2), 235-243.

Newbold, P. (1990). "Precise and Efficient Computation of Beveridge-Nelson Decomposition of Economic Time Series", J ournal of M onetary E conomics, 26, 453-457

Ord, J .K., A. B. Koehler, and R. D. Snyder (1997). "Estimation and Prediction for a Class of Dynamic Nonlinear Statistical Models", J ournal of the American Statistical A ssociation, Vol .92, No.440, 1621-1629.

Snyder, R. D. (1985). "Recursive Estimation of Dynamic Linear Models", J ournal of the Royal Statistical Society, series B, 47, 272-276. 
Stock, J. H., and M. W. Watson (1988). "Variable Trends in Economic Time Series", J ournal of E conomic Perspectives, Vol 2(3), 147-174.

Watson, M. W. (1986). "Univariate Detrending Methods with Stochastic Trends", Journal of M onetary E conomics, 18, 49-75. 